\title{
Cost Impact of Bevacizumab and Cetuximab Associated Therapies in Colorectal Cancer in Veneto Region
}

\author{
Simonetta Ballali ${ }^{1}$, Daniele Chiffi ${ }^{2}$, Marta P. Trojniak ${ }^{3}$, Dario Gregori ${ }^{*}, 2$ and the EIHCD-VeRo \\ Research Project Agreement ${ }^{\S}$
}

\author{
${ }^{I}$ Prochild Onlus, Trieste, Italy \& Unit of Biostatistics, Epidemiology and Public Health, Department of Cardiac, \\ Thoracic and Vascular Sciences, University of Padova, Padova, Italy \\ ${ }^{2}$ Unit of Biostatistics, Epidemiology and Public Health, Department of Cardiac, Thoracic and Vascular Sciences, \\ University of Padova, Padova, Italy \\ ${ }^{3}$ Oncology Pharmacy Department, Istituto Oncologico Veneto, IRCCS, Padova, Italy
}

\begin{abstract}
Objective: To assess the economic impact of the introduction of bevacizumab and cetuximab, in 1 st and $2 \mathrm{nd}$ line treatment of $\mathrm{mCRC}$ patients in Veneto region (North-East of Italy).

Methods: A Markov state decision model was implemented to evaluate the cost impact of bevacizumab and cetuximab use in patients with mCRC for a lapse of time of three years in Veneto public hospitals. The Markov model expressed transition probabilities from three different states, comparing in addition the expected deaths and the monthly survival rates in treatment and no-treatment groups, along the lines of previously published studies.

Results: The cost impact of bevacizumab administration in patients with mCRC accounted a mean value of $18268788 €$ within the first 6 months. Cetuximab therapy for those refractory to $1^{\text {st }}$ line treatment, increased costs of almost $833340 €$ in the first 6 months, increasing in the following period due to a higher portion of patients switching from a stable status to a progressive one.

Discussion: The cost impact of monoclonal antibodies on health expenses is very high. For a regional cohort like the Veneto's one, figure sets around $19000000 €$ in 6 months, when considering $1^{\text {st }}$ and $2^{\text {nd }}$ line treatment, reaching the level of $50000000 €$ within three years.
\end{abstract}

Keywords: Bevacizumab, cetuximab, cost, KRAS wild type, metastatic colorectal cancer, Veneto.

\section{INTRODUCTION}

At the present time, colorectal cancer (CRC) is the third most frequent cancer [1]. Worldwide, colorectal cancer represents $9.4 \%$ of all incident cancer in men and $10.1 \%$ in

*Address correspondence to this author at the Unit of Biostatistics, Epidemiology and Public Health, Department of Cardiac, Thoracic and Vascular Sciences, Via Loredan 18, 35121 Padova, Italy; Tel: +39 049 8275384; Fax:+3902 700445089; E-mail: dario.gregori@unipd.it

${ }^{\S}$ EIHCD-VeRo (Economic Impact of High Cost Drugs in the Veneto Region) Research Project Agreement members: Dario Gregori (Principal Investigator), Unit of Biostatistics, Epidemiology and Public Health, Department of Cardiac, Thoracic and Vascular Sciences, University of Padova, Padova, Italy; Daniele Chiffi, Unit of Biostatistics, Epidemiology and Public Health, Department of Cardiac, Thoracic and Vascular Sciences, University of Padova, Padova, Italy; Francesco Grigoletto, Department of Environmental Medicine and Public Health, University of Padova, Italy; Egle Perissinotto, Unit of Biostatistics, Epidemiology and Public Health, Department of Cardiac, Thoracic and Vascular Sciences, University of Padova, Padova, Italy; Alessandra Buja, Department of Molecular Medicine, University of Padova, Italy; Andrea Tramarina, Veneto Regional Health Agency, Italy; Costantino Gallo, Veneto Regional Health Agency, Italy; Antonio Compostella, Veneto Regional Health Agency, Italy; Davide Pastorelli, Istituto Oncologico del Veneto IRCSS, Padova, Italy; Giuseppe Rausa, Department of Environmental Medicine and Public Health, University of Padova, Italy. women [2]. Metastases are already present at the time of diagnosis in $20-25 \%$ and $50-60 \%$ most likely will develop metastases [3].

In the majority of cases, patients with metastatic CRC (mCRC) are enrolled for palliative care treatments, rather than in curative protocols. Therapeutic approach, mostly second and third line approach aims instead at reducing metastasis size, in order to give the opportunity to eradicate them through surgery [4].

Bevacizumab is a humanized monoclonal antibody against vascular endothelial growth factor (VEGF) introduced as first line treatment, resulting in prolonging overall survival and progression free survival [5-7]. Bevacizumab is approved as therapeutic regimen by Food and Drug Administration (FDA) and European Medicines Agency (EMA) in combination with fluoropyrimidine based chemotherapy [8].

Cetuximab was approved for the treatment of $\mathrm{mCRC}$ in 2004 [9], and it entered in recommendations for the use of monoclonal antibody therapy around 2008 [10, 11]. As complement of chemotherapy, cetuximab works as second line agent, targeting epidermal growth factor receptors (EGFR), contributing to reduce liver metastases to an 
operable size, which can lead to the complete recovery of the patient. The main limitation is the peculiarity of actions towards mCRC expressing V-Ki-ras2 Kirsten rat sarcoma viral oncogene homolog (KRAS).

The constant increase in health care costs indicates that by 2020 the total Italian health care expenditures will account for growing proportions of the gross domestic product. High cost drugs in particular, targeted therapies such as bevacizumab and cetuximab, have been highly studied to assess the exact target population, in order to minimize the societal impact of their use. Using the treatment of colorectal cancer with bevacizumab as an example, it is commonly stated that more information is needed to properly evaluate the implication of high cost drugs with their proven benefit. In this scenario, advances in the treatment of CRC in both the adjuvant and metastatic settings have presented significant financial implications for health-care systems in economically developed countries at national and regional level $[12,13]$.

The present paper aims at assessing the economic impact of the use of monoclonal antibodies targeting mCRC in public hospitals of Veneto region, North-East of Italy.

\section{MATERIALS AND METHODS}

\section{Antibody Therapy}

Bevacizumab is the first anti-angiogenic drug to be used in mCRC [14]. Bevacizumab, produced by incorporating six VEGF-binding residues from a murine anti-human VEGF monoclonal antibody into a human IgG framework, binds to soluble VEGF and prevents it from binding to its receptors, VEGFR-1 or VEGFR-2, predominantly on vascular endothelial cells [15]. The actions of VEGF-A, mediated principally via the VEGF receptors 1 and 2, include the regulation of vascular permeability and neovascularisation [14]. Bevacizumab is currently approved in the USA and EU for the treatment of $\mathrm{mCRC}$ in combination with first-line 5Fluoropyrimidine-based chemotherapy, like FOLFOX regimen (leucovorin, 5-fluorouracil (5-FU) and oxaliplatin) or FOLFIRI regimen (leucovorin, 5-FU and irinotecan). The overall impact of bevacizumab on survival of patients with this disease has been difficult to quantify, although it seems probable that its use throughout the period of chemotherapy may maximize the benefit. The recommended dose of bevacizumab is $5 \mathrm{mg} / \mathrm{kg}$ of body weight every two weeks in association with FOLFOX or FOLFIRI.

The chimeric IgG1 monoclonal antibody cetuximab was the first biologic directed against EGFR to receive approval by the FDA for use in mCRC. Cetuximab binds specifically to the extracellular domain of EGFR as a competitive antagonist of the endogenous ligands augmenting as tumor growth inhibition [16], especially when associated with cytotoxic agents like topotecan, and more significantly, with irinotecan. The cetuximab-irinotecan combination produces moreover growth inhibition of irinotecan-refractory DLD-1 and HT-29 xenografts, whereas tumor growth was not controlled by either agent alone. It has been widely proven that the addition of cetuximab to FOLFIRI has improved the life expectancy of patients with $\mathrm{mCRC}$ by almost 2 years [17]. The benefit of anti-EGFR antibodies in mCRC has been found to be limited to patients with KRAS wild-type tumor [18]. KRAS gene encodes a small G protein that links ligand-dependent receptor activation to intracellular pathway of the EGFT signaling cascade. Mutation of key sites within the gene causes constitutive activation of KRAS associated signaling, leading to an inefficient action of cetuximab. Cetuximab is given weekly, in association with irinotecan, with a loading dose of $400 \mathrm{mg} / \mathrm{m}^{2}$ body surface in the first week, followed by a regular administration of $250 \mathrm{mg} / \mathrm{m}^{2}$ of body surface. All data are presented in Table $\mathbf{1}$.

\section{Setting}

Veneto is an Italian region located in the North East with a population of 4937854 (year 2010) [19] and the incidence of new cases of colorectal cancer in Veneto is greater than 3000 cases per year [20].

\section{Target Population}

The determination of the target population for bevacizumab was obtained by taking into account the annual

Table 1. Characteristics of Monoclonal Antibodies Used in Metastatic Colorectal Cancer (BW, Body Weight; BS, Body Surface)

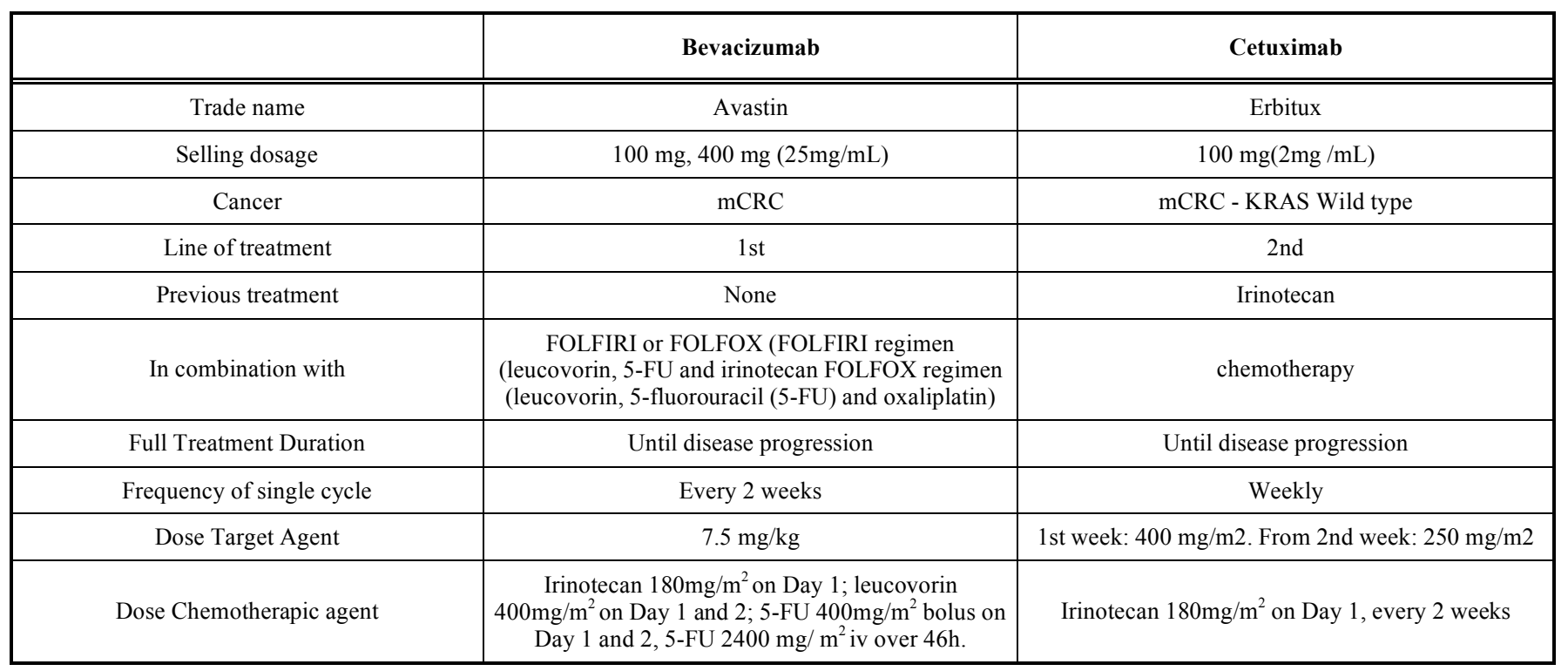


incidence of $\mathrm{mCRC}$ patients, the criteria of clinical appropriateness, the yearly incidence rates of people with mCRC at diagnosis, and the proportion of patients which were not eligible for receiving bevacizumab treatment because of side effects. All data were obtained from published studies $[3,6,9,18,21]$. Data on incidence and prevalence of mCRC were obtained from the pharmacological database of IOV (Veneto Institute for Oncology) [20].

Cetuximab's target population was calculated considering the yearly probabilities to be a first line refractory and bearing KRAS wild- type (Table 4). All data were obtained from SISTAR website [19] and from major published studies $[3,6,18]$.

\section{Markov Model}

A Markov state decision model was selected to evaluate the cost impact of bevacizumab and cetuximab use for a lapse of time of two years in Veneto public hospitals, considering transition probabilities from three different states, and by comparing the expected deaths and the monthly survival rates in treatment and no-treatment groups (Fig. 1). This indicator has already been proven to be valid tool for costs considerations [22]. Markov Model consists of 3 different states: "Stable disease", "Progressive disease", and "Dead". Patients started in the state "Stable disease" where they receive treatment. In case of Cetuximab, eligible patients present no KRAS mutation (wild-type). Patients could subsequently respond to the therapy and remain in the "stable state" or transit to the state "Progressive disease" or

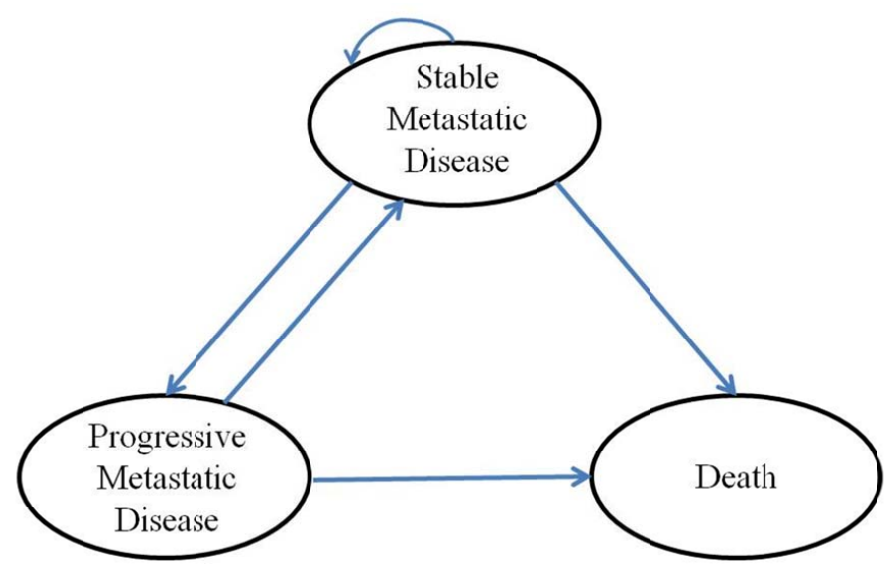

Fig. (1). Markov model.

Table 2. Incidences and Probabilities of Transition Among Markov's Model Status in Patients Not Receiving Bevacizumab as $1^{\text {st }}$ Line Therapy

\begin{tabular}{|c|c|c|c|c|c|c|c|c|c|c|c|}
\hline 密 & 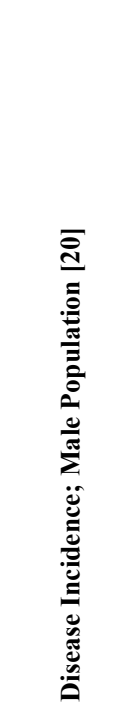 & 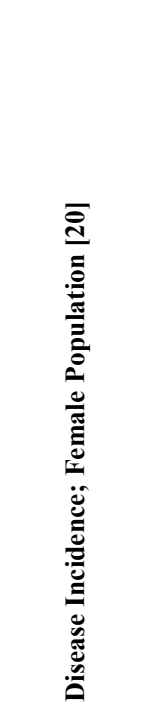 & 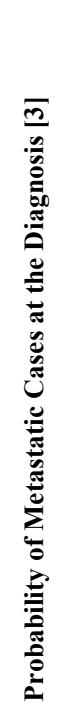 & 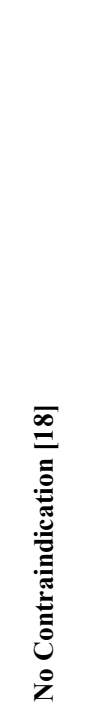 & 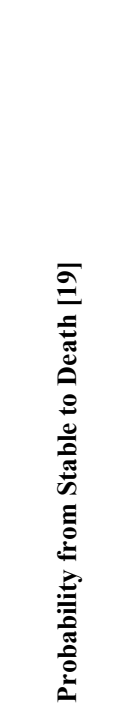 & 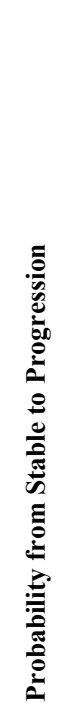 & 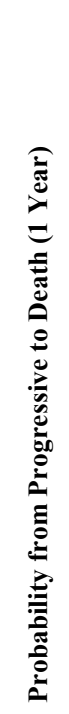 & 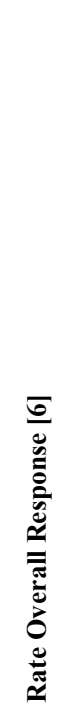 & 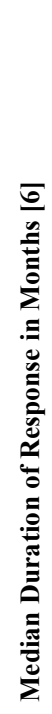 & 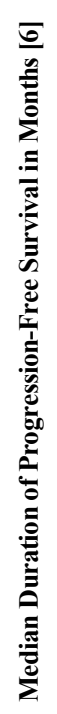 & 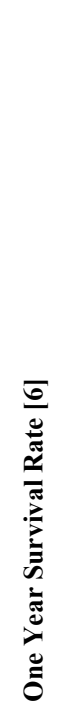 \\
\hline $0-24$ & 0.0000026 & 0.0000026 & 0.25 & 0.975 & 0.0006761 & 0.42 & 0.37 & 0.35 & 7.1 & 6.2 & 0.63 \\
\hline $25-49$ & 0.0001124 & 0.0001142 & 0.25 & 0.975 & 0.0008002 & 0.42 & 0.37 & 0.35 & 7.1 & 6.2 & 0.63 \\
\hline $50-74$ & 0.0020598 & 0.001219 & 0.25 & 0.975 & 0.0085 & 0.42 & 0.37 & 0.35 & 7.1 & 6.2 & 0.63 \\
\hline$\geq 75$ & 0.0051986 & 0.00282966 & 0.25 & 0.975 & 0.011 & 0.42 & 0.37 & 0.35 & 7.1 & 6.2 & 0.63 \\
\hline
\end{tabular}


to the state "Dead". Patients in the "Progressive State" could once again respond to the therapy, remain in the same state or switch to the "Death state". Death due to colorectal cancer was considered as possible only for patients in the state "Progressive disease", otherwise the transition to "Dead" was due to other causes. The reference population mimicked the distribution of the Veneto population, assuming a median drug dosage for a person of $60 \mathrm{kgs}$. The transition probabilities for clinical variables are shown in Tables 2-4.

Transition probabilities (reflecting the 6-month cycle length) for bevacizumab and cetuximab and for people receiving only chemotherapy were derived from major

Table 3. Incidences and Probabilities of Transition Among Markov's Model Status in Patients Receiving Bevacizumab as $1^{\text {st }}$ Line Therapy

\begin{tabular}{|c|c|c|c|c|c|c|c|c|c|c|c|}
\hline$\underset{\&}{8}$ & 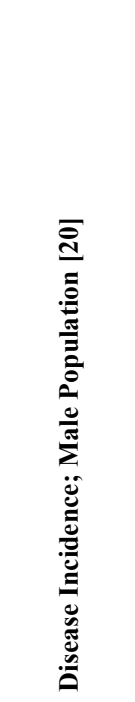 & 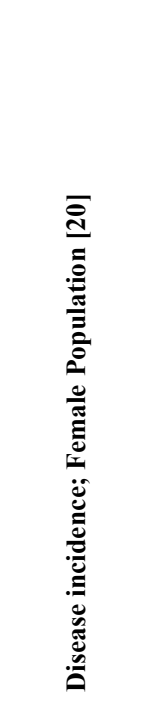 & 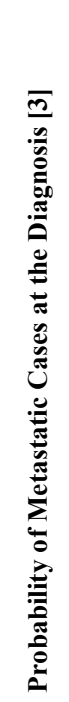 & 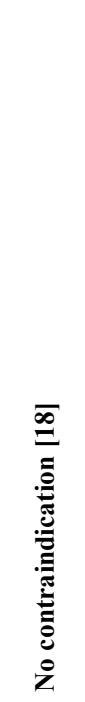 & 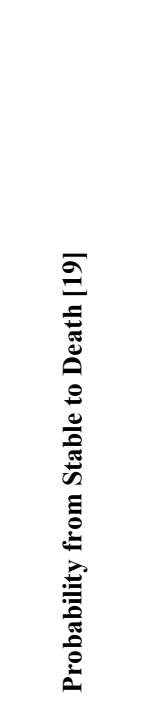 & 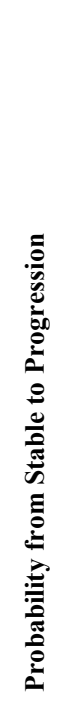 & 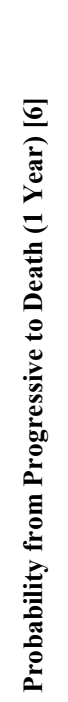 & 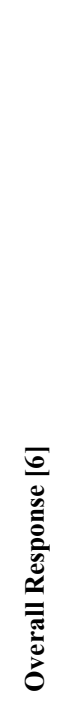 & 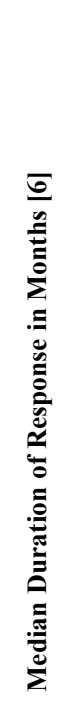 & 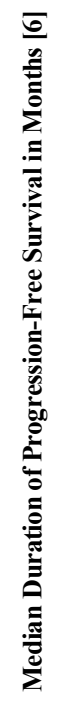 & 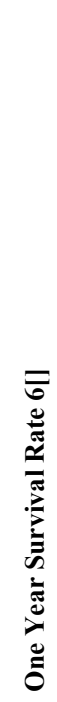 \\
\hline $0-24$ & 0.0000026 & 0.0000026 & 0.25 & 0.975 & 0.0006761 & 0.29 & 0.26 & 0.45 & 10.4 & 10.6 & 0.74 \\
\hline $25-49$ & 0.0001124 & 0.0001142 & 0.25 & 0.975 & 0.0008002 & 0.29 & 0.26 & 0.45 & 10.4 & 10.6 & 0.74 \\
\hline $50-74$ & 0.0020598 & 0.001219 & 0.25 & 0.975 & 0.0085 & 0.29 & 0.26 & 0.45 & 10.4 & 10.6 & 0.74 \\
\hline$\geq 75$ & 0.0051986 & 0.00282966 & 0.25 & 0.975 & 0.011 & 0.29 & 0.26 & 0.45 & 10.4 & 10.6 & 0.74 \\
\hline
\end{tabular}

Table 4. Incidences and Probabilities of Transition Among Markov's Model Status in Patients Receiving Cetuximab as $2^{\text {nd }}$ Line Therapy

\begin{tabular}{|c|c|c|c|c|c|c|c|c|c|c|c|c|c|c|}
\hline 选 & 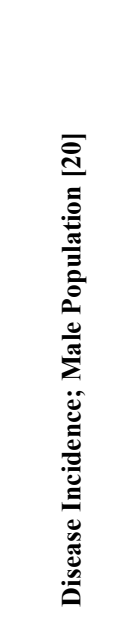 & 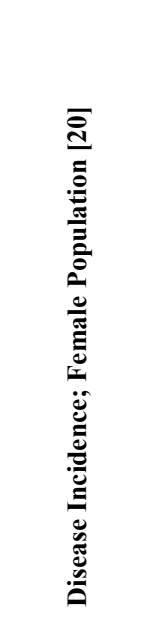 & 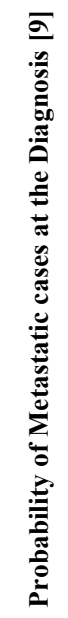 & 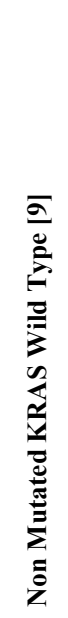 & 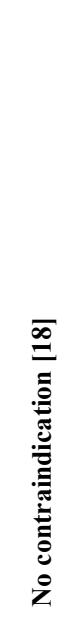 & 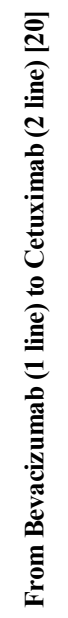 & 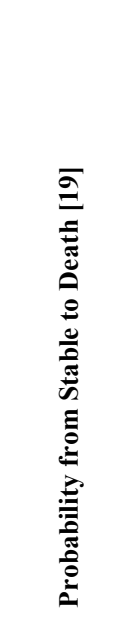 & 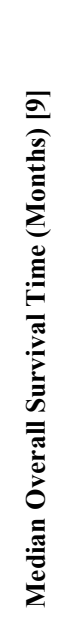 & 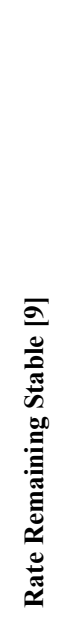 & 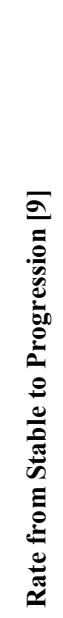 & 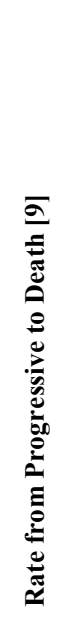 & 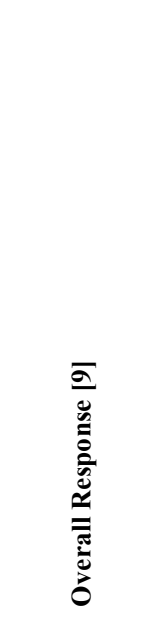 & 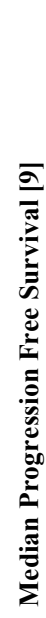 & 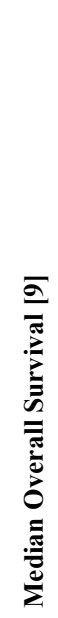 \\
\hline $0-24$ & 0.0000026 & 0.0000026 & 0.25 & 0.64 & 0.975 & 0.57 & 0.0006761 & 19.9 & 0.37 & 0.49 & 0.40 & $0.429-0.510$ & 8.9 & 19.9 \\
\hline $25-49$ & 0.0001124 & 0.0001142 & 0.25 & 0.64 & 0.975 & 0.57 & 0.0008002 & 19.9 & 0.37 & 0.49 & 0.40 & $0.429-0.510$ & 8.9 & 19.9 \\
\hline $50-74$ & 0.0020598 & 0.001219 & 0.25 & 0.64 & 0.975 & 0.57 & 0.0085 & 19.9 & 0.37 & 0.49 & 0.40 & $0.429-0.510$ & 8.9 & 19.9 \\
\hline$\geq 75$ & 0.0051986 & 0.00282966 & 0.25 & 0.64 & 0.975 & 0.57 & 0.011 & 19.9 & 0.37 & 0.49 & 0.40 & $0.429-0.510$ & 8.9 & 19.9 \\
\hline
\end{tabular}


published studies $[3,6]$, necessary for the drug approval of FDA, EMA and institutional-derived data from IOV [20]. Monthly transition probabilities were determined from first year clinical probabilities, using the following formula:

$P=1-e^{-r t}$

where $P$ is the probability of the event, $e$ is the natural logarithm, $r$ is the rate, and $t$ is the time interval [23].

Data were divided into three categories, patients undergoing only regular chemotherapy treatment with FOLFIRI as $1^{\text {st }}$ line treatment (bevacizumab -), patients receiving bevacizumab in association with FOLFIRI as $1^{\text {st }}$ line treatment (bevacizumab + ), patients receiving cetuximab as $2^{\text {nd }}$ line therapy (cetuximab +). All data are presented in Tables 2-4. FOLFIRI was chosen as chemotherapic protocol associated with bevacizumab administration taken its common use in Veneto's hospital [20].

The costs were related to the cost of the drug plus the costs of the chemotherapeutic agents, when given in association (Table 1). Three scenarios were simulated in order to determine the economic impact: for $1^{\text {st }}$ line treatment, one scenario considered the patients receiving both chemotherapy and bevacizumab and the other one in which patients only received chemotherapy. The third scenario considered eligible patients receiving cetuximab as $2^{\text {nd }}$ line treatment after the failure of $1^{\text {st }}$ line treatment with bevacizumab+FOLFIRI.

The authors stated that they took an Italian National Healthcare Service (NHS) perspective.

\section{Simulations}

A micro-simulation approach has been used as the main setting for the analysis. All quantities described above have been implemented in the stochastic simulation model as expected values of suitable probability functions. More in detail, for discrete random variables (e.g.: number of people incident for mCRC), a binomial model has been used (e.g.: sampling from the Veneto population with probability equal to the age-specific incidence rate). For continuous random variables (e.g.: weight of patients for drug dosage administration), including costs, a Kumaraswamy distribution, which is a very flexible both as symmetric and asymmetric twoparameters distribution, has been used [24]. Thus, 10000 Monte Carlo runs have been performed, deriving the empirical distributions of the target quantities of interest (e.g.: cost of care), for which selected summary measures have been computed (e.g.: mean, $5^{\text {th }}$ and $95^{\text {th }}$ percentile, to be used as $90 \%$ credibility intervals for inferential purposes). All estimated quantities are reported along with $90 \%$ credibility intervals. Software used for simulations was the VOSE Model Risk analyzer [25].

\section{RESULTS}

Bevacizumab as $1^{\text {st }}$ line therapy showed a significant impact on death rate in the first 2 years considered in the analysis (Table 5). Patients undergoing bevacizumab treatment showed a higher overall survival compared to those receiving only FOLFIRI therapy, as presented in Table 5. Cetuximab was considered as $2^{\text {nd }}$ line therapy only in patients with KRAS wild type tumor, and it showed a positive impact in the first year period, decreasing the number of patients switching from a stable state to a progressive one. The cost impact of bevacizumab administration in patients with mCRC accounted a mean value of $18268788 €$ within the first 6 months. Cetuximab therapy for those refractory to $1^{\text {st }}$ line treatment, increased costs of almost $833340 €$ in the first 6 months, increasing in the following period due to a higher portion of patients switching from a stable status to a progressive one. Data on costs are presented in Table 6. All costs are given as sum of the cost of the monoclonal antibody plus the chemotherapic agent associated.

\section{DISCUSSION}

In order to understand the impact that mCRC treatments have on the considered health care system, a brief description of Italian and regional health system needs to be introduced. Health care expenditures have progressively increased over time, and a central containment has been required in all the European Union. During the late 1990s, Italy's administrative and institutional settings started to become those of a federal state [13]. In 1997, social insurance contributions were cut out and the regionally collected system of tax financing was implemented, with general taxation covering an ancillary role. The chronic regional deficit is nowadays an systematic reality [26]. In the latest years, an increase in expenditure was registered, mostly due to drugs expenses, raised due to the loss of copayment revenue and to an increased consumption. When considering recent data on total health care expenditures at the regional level, Veneto is the second region devoting a greater than average amount of resources to hospital care, $47.4 \%$ of the total and one of those regions that have experienced a significant decrease in regional budget deficit after the new reforms at the beginning of this century. In Italy, reimbursement decisions for cancer drugs are taken by one regulatory body, the National Health System (Sistema Sanitario Nazionale, SSN) in consultation with Agenzia Italiana del Farmaco (AIFA). Taken that in Italy almost all high cost anti-cancer drugs are listed as Class $\mathrm{H}$ drugs, significant savings can be achieved due to the fact that drugs bought directly by hospitals are granted a minimum $50 \%$ discount by pharmaceutical companies [13].

In 2010, formal indications were accepted for 10 cancer drugs, among those bevacizumab and cetuximab, by the licensing authorities of 13 countries: Australia, Canada (Ontario), England, Finland, France, Italy, Germany, Japan, New Zealand, the Netherlands, Scotland, Sweden, and the United States (Medicare Parts B and D) [12]. In Italy, bevacizumab and cetuximab are currently used as $1^{\text {st }}$ and $2^{\text {nd }}$ line therapeutic target agents in mCRC treatment.

Bevacizumab use has been widely explored. The mechanism of its action is complex and unlikely to be limited to the inhibition of tumor angiogenesis. Postulated actions include the induction of epithelial cell apoptosis, sensitizing tumor endothelial cells to chemotherapy-induced apoptosis, inhibition of the VEGF mediated mobilization of endothelial progenitor cells, vasoconstriction via inhibition of nitric oxide and prostacyclin, normalization of tumor vasculature allowing improved chemotherapy and oxygen delivery, 
Table 5. Impact of Regular Therapy and Monoclonal Antibodies Use on Overall Survival and Progression of mCRC Patients of Veneto's Cohort. All Data are Presented with a 90\% Credibility Interval (C.I.)

\begin{tabular}{|c|c|c|c|}
\hline & $\begin{array}{c}\text { Pts Receiving Bevacizumab as 1st } \\
\text { Line Treatment }\end{array}$ & $\begin{array}{c}\text { Pts Not Receiving Bevacizumab as } \\
\text { 1st Line Treatment }\end{array}$ & $\begin{array}{c}\text { Pts Receiving Cetuximab as 2nd } \\
\text { Line Treatment }\end{array}$ \\
\hline \hline Eligible at the beginning & $1414(1352 ; 1476)$ & $1414(1352 ; 1476)$ & Not applicable \\
\hline Stable at the beginning & $898(845 ; 952)$ & $738(691 ; 788)$ & $857(789 ; 926)$ \\
\hline Eligible in 36 months & Not applicable & Not applicable & $40(30 ; 50)$ \\
\hline in Progression by the 6th month & $411(375 ; 446)$ & $456(419 ; 493)$ & $257(230 ; 285)$ \\
\hline in Progression by the 12th month & $349(318 ; 381)$ & $340(309 ; 372)$ & $194(170 ; 218)$ \\
\hline in Progression by the 18th month & $249(223 ; 277)$ & $55(43 ; 67)$ & $88(73 ; 104)$ \\
\hline in Progression by the 24th month & $127(108 ; 145)$ & $5(2 ; 9)$ & $21(13 ; 28)$ \\
\hline in Progression by the 30th month & $33(24 ; 42)$ & $0(0 ; 0)$ & $1(0 ; 3)$ \\
\hline in Progression by the 36th month & $2(0 ; 5)$ & $218(195 ; 243)$ & $102(85 ; 118)$ \\
\hline Dead by the 6th month & $105(88 ; 122)$ & $531(490 ; 572)$ & $167(145 ; 189)$ \\
\hline Dead by the 12th month & $308(279 ; 339)$ & $933(878 ; 992)$ & $390(354 ; 425)$ \\
\hline Dead by the 18th month & $626(581 ; 671)$ & $1271(1201 ; 1340)$ & $610(561 ; 658)$ \\
\hline Dead by the 24th month & $1013(955 ; 1073)$ & $1401(1328 ; 1475)$ & $708(654 ; 763)$ \\
\hline Dead by the 30th month & $1311(1242 ; 1380)$ & $1413(1340 ; 1488)$ & $729(673 ; 785)$ \\
\hline Dead by the 36th month & $1407(1334 ; 1479)$ & & \\
\hline
\end{tabular}

Table 6. Cost Impact of Monoclonal Antibodies in Association with Chemotherapy in mCRC $1^{\text {st }}$ and $2^{\text {nd }}$ Line Treatments. Cetuximab Costs has been Considered as Cumulative with Previous 1st Line Treatments. All Costs are Given with a $90 \%$ C.I.

\begin{tabular}{|c|c|c|}
\hline & Bevacizumab & Bevacizumab + Cetuximab \\
\hline \hline Cumulative cost at 6th month & $18268788(12397543 ; 22537051)$ & $19102128(13239943 ; 23382259)$ \\
\hline Cumulative cost at 12th month & $33644438(22784157 ; 41429600)$ & $34842409(24067447 ; 42648904)$ \\
\hline Cumulative cost at 18th month & $44595920(30225347 ; 54838465)$ & $46125721(31836700 ; 56433155)$ \\
\hline Cumulative cost at 24th month & $50155178(34012782 ; 61758551)$ & $51764949(35744637 ; 63396066)$ \\
\hline Cumulative cost at 30th month & $51586838(35018508 ; 63535257)$ & $53199568(36702742 ; 65155422)$ \\
\hline Cumulative cost at 36th month & $51681639(35099974 ; 63619739)$ & $53294374(36764375 ; 65304174)$ \\
\hline
\end{tabular}

immune modulation via inhibition of dendritic cell function, counteracting chemotherapy and radiotherapy-induced VEGF signaling and direct effects on tumor cells [27]. Hurwitz and colleagues studied the addition of bevacizumab to bolus irinotecan, fluorouracil, and leucovorin (IFL) in patients with previously untreated mCRC, showing a clinically meaningful and statistically significant improvement in overall survival, progression-free survival, and response rate [6].

Once measured the significant efficacy of the considered drug, the economic impact on regional health care budget needs therefore to be evaluated, taken that as costs rise, countries have opted to control utilization by restricting offlabel use or limiting reimbursement to subpopulations with the greatest benefits as determined by cost-effectiveness analysis (CEA) [12].

Cetuximab in combination with irinotecan is currently indicated from AIFA for the treatment of mCRC expressing
EGFR after failure of the citotoxic therapy based on solo irinotecan, improving life expectancy of treated patients, whose metastasis are viable for surgery after the reduction obtained through therapy. The addition of cetuximab results in a significant improvement in overall survival time [17], although this success remains limited to the patients with KRAS wild-type disease.

Patients undergoing this treatment are therefore given the chance of short-term survival or recovery.

Survival increases as patients with $\mathrm{mCRC}$ are exposed to all available agents, but this benefit comes at high cost. This cost might exceed common thresholds of regional health service institutions.

As shown from our results, the cost impact of monoclonal antibodies on health expenses is very high. For a regional cohort like the Veneto's one, figure sets around $19000000 €$ when considering $1^{\text {st }}$ and $2^{\text {nd }}$ line treatment, 
reaching the level of $50000000 €$ within three years. A careful planning of patients' eligibility is therefore recommended, taken the proven efficacy of those antibodies on $\mathrm{mCRC}$.

These results were similar to those retrieved in Greece's scenario, analyzed in Fragoulakis' paper [28], that considered just Bevacizumab's costs. From the NHS perspective, the mean total costs per patient were slightly higher than the Greek ones $(€ 22,382$ against $€ 19,701)$. In any case, in a Markov model submitted to the National Institute for Clinical Excellence by the manufacturer of cetuximab, cetuximab seemed to be a cost-effective choice compared with other drugs [29]. The model included individuals with EGFR-expressing Kirsten rat sarcoma viral oncogene homolog wild-type mCRC who had received second- or subsequent-line chemotherapy for metastatic disease and had a 10-year time horizon and considered the NHS perspective.

When comparing these costs of mCRC treatments with those necessary for the screening of the same pathology, then some evidences can be presented.

In Italy there are three formal cancer screening programs: breast, cervical and CRC screening. Breast and cervical cancer screenings are more widely spread than CRC screening due to their earlier introduction. Regional differences are reported for all three programs.

In persons over 85 years, CRC constitutes one third of all neoplasms with $70 \%$ of patients aged 65 years or older [30]. Particularly, in persons $>65$ years old, the incidence of colon cancer is about 120 new cases per 100,000 inhabitants per year (and in persons $>75$ years old is about 200/100,000 inhabitants per year) and mortality is about 90 per 100,000 inhabitants per year. Since, an estimated 90/100,000 inhabitants per year CRC deaths are expected to occur in patients older than 65 and since the majority of these would be aged 65 or older $(65-78 \%$ CRC), there could be a substantial potential for population benefit with CRC screening [31].

Bracci and Pizzo estimated that for the city of Ferrara the overall cost related to the introduction of a CRC screening programe was approximately $€ 1,400,000$ (from October 2005 until March 2007 with more than 99,000 individuals invited) with a large proportion of these costs related to the implementation and management of the programe [32].

As highlighted in the report on CRC issued by the Osservatorio Nazionale (National Observatory) on Screening in 2011 [33], among screening campaigns, CRC screening has shown the greatest growth, although present Italian economic crisis might reflect in significant reductions of budget for regional health. Within the 2001 financial budget [34], it was decided that in the target population (older than 45 years old or population at risk) screening was free of charge. In 2004 the Ministry of Health (Ministero della Salute) redistributed overall $€ 7,000,000$, a minimum of $€$ 50,000 per region, for reducing the gaps in cancer screenings and activating the CRC screening programe $(€ 1,750,000$ specifically for CRC screening) [35]. With the 2005 stateregional agreement, further specific funds were allocated to enhance the spread and coverage of cancer screenings. The cost of screening is typically less than the cost of treating cancer. When screening identifies a colorectal tumor in its early stages, the cost of treatment is often much less expensive than if the tumor is detected later in the course of disease [36].

The adopted screening programes in Italy employ the faecal occult blood test (FOBT), while, nearly restricted to Piemonte region, some regions have adopted flexible sigmoidoscopy (FS) once in a lifetime and FOBT for nonresponders to FS [37].

When considering screening diffusion, Veneto region showed a theoretical extension of screening programs' coverage of $94.3 \%$ in 2009, in residents 50-69 years old [37], representing the region with the highest extension in the whole country. Real situation, considered as the data on adjusted compliance, calculated as the proportion of subjects invited to attend screening (minus those with a wrong address and those excluded after invitation for a recent test) who underwent a screening test, Veneto region sets again among the first positions, showing a mean compliance of $66.9 \%$. From a recent report on CRC screening, the average cost per FS examined person was of $85 €$ per person, reaching $95 €$ when adding histological examination of colorectal polyps [38]. The costs for the FOBT vary instead from $11 €$ to $20 €$ in a 10 year period [38].

As considered from WHO, $40 \%$ of cancers could be avoided through prevention, while $40 \%$ could be cured (if detected early) [39]. When compared with prevention costs, cost impact of adjuvant therapies in Veneto appeared way higher, even when considering just first line therapy. Taken the high economic impact of these drugs on regional health system, a careful evaluation of eligible patients needs to precede every prescription, while a broader screening campaign might be implemented in order to contain costs of late tumor detection.

\section{CONFLICT OF INTEREST}

The authors certify that there is no conflict of interest with any organization regarding the material discussed in the manuscript.

\section{ACKNOWLEDGEMENTS}

This research was partially funded by an unrestricted grant of the University of Padova, Progetti di Ateneo 2009, (Code CPDA093183/09, "Modello per la valutazione dell'impatto economico, sociale e di salute derivante dall'utilizzo dei nuovi farmaci oncologici nella regione Veneto").

\section{REFERENCES}

[1] Ferlay, J.; Shin, H. R.; Bray, F.; Forman, D.; Mathers, C.; Parkin, D. M. Estimates of worldwide burden of cancer in 2008: GLOBOCAN 2008. Int. J. Cancer, 2011, 127 (12), 2893-2917.

[2] Boyle, P.; Langman, J. S. ABC of colorectal cancer: Epidemiology. BMJ, 2000, 321 (7264), 805-808.

[3] Van, Cutsem, E.; Oliveira, J. Advanced colorectal cancer: ESMO clinical recommendations for diagnosis, treatment and follow-up. Ann. Oncol., 2009, 20 Suppl 4, 61-63.

[4] Folprecht, G.; Grothey, A.; Alberts, S.; Raab, H. R.; Kohne, C. H. Neoadjuvant treatment of unresectable colorectal liver metastases: correlation between tumour response and resection rates. Ann. Oncol., 2005, 16 (8), 1311-1319.

[5] Giantonio, B. J.; Catalano, P. J.; Meropol, N. J.; O'Dwyer, P. J.; Mitchell, E. P.; Alberts, S. R.; Schwartz, M. A.; Benson, A. B. 3rd, Bevacizumab in combination with oxaliplatin, fluorouracil, and leucovorin (FOLFOX4) for previously treated metastatic colorectal 
cancer: results from the Eastern Cooperative Oncology Group Study E3200. J. Clin. Oncol, 2007, 25 (12), 1539-1544.

[6] Hurwitz, H.; Fehrenbacher, L.; Novotny, W.; Cartwright, T.; Hainsworth, J.; Heim, W.; Berlin, J.; Baron, A.; Griffing, S.; Holmgren, E.; Ferrara, N.; Fyfe, G.; Rogers, B.; Ross, R.; Kabbinavar, F. Bevacizumab plus irinotecan, fluorouracil, and leucovorin for metastatic colorectal cancer. N. Engl. J. Med., 2004, 350 (23), 2335-2342.

[7] Kabbinavar, F. F.; Hurwitz, H. I.; Yi, J.; Sarkar, S.; Rosen, O. Addition of bevacizumab to fluorouracil-based first-line treatment of metastatic colorectal cancer: pooled analysis of cohorts of older patients from two randomized clinical trials. J. Clin. Oncol., 2009, 27 (2), 199-205.

[8] Edwards, M. S.; Chadda, S. D.; Zhao, Z.; Barber, B. L.; Sykes, D. P. A systematic review of treatment guidelines for metastatic colorectal cancer. Colorectal. Dis, 2012, 14 (2), e31-47.

[9] Van Cutsem, E.; Kohne, C. H.; Hitre, E.; Zaluski, J.; Chang Chien, C. R.; Makhson, A.; D'Haens, G.; Pinter, T.; Lim, R.; Bodoky, G.; Roh, J. K.; Folprecht, G.; Ruff, P.; Stroh, C.; Tejpar, S.; Schlichting, M.; Nippgen, J.; Rougier, P. Cetuximab and chemotherapy as initial treatment for metastatic colorectal cancer. N. Engl. J. Med., 2009, 360 (14), 1408-1417.

[10] Van Cutsem, E. J.; Oliveira, J. Advanced colorectal cancer: ESMO clinical recommendations for diagnosis, treatment and follow-up. Ann. Oncol., 2008, 19 Suppl, 2, ii33-34.

[11] Colon cancer: ESMO clinical recommendations for diagnosis, adjuvant treatment and follow-up. Ann. Oncol., 2008, 19 Suppl 2, ii29-30.

[12] Cheema, P. K.; Gavura, S.; Migus, M.; Godman, B.; Yeung, L.; Trudeau, M. E. International variability in the reimbursement of cancer drugs by publically funded drug programs. Curr. Oncol., 2012, 19 (3), e165-176.

[13] Lo Scalzo, A.; Donatini, A.; Orzella, L.; Cicchetti, A.; Profili, S.; Maresso, A. Italy: health system review Health. Syst. Transit, [Online].2009, p.1-126. Available at: http://www.euro.who.int/dd ata/assets/pdf_file/0006/87225/E93666.pdf [Accessed on: $19^{\text {th }}$ July 2012].

[14] Okines, A.; Cunningham, D. Current perspective: bevacizumab in colorectal cancer--a time for reappraisal? Eur. J. Cancer, 2009, 45 (14), 2452-2461.

[15] McCormack, P. L.; Keam, S. J. Bevacizumab: a review of its use in metastatic colorectal cancer. Drugs, 2008, 68 (4), 487-506.

[16] Overholser, J. P.; Prewett, M. C.; Hooper, A. T.; Waksal, H. W.; Hicklin, D. J. Epidermal growth factor receptor blockade by antibody IMC-C225 inhibits growth of a human pancreatic carcinoma xenograft in nude mice. Cancer, 2000, 89 (1), 74-82.

[17] Van Cutsem, E.; Kohne, C. H.; Lang, I.; Folprecht, G.; Nowacki, M. P.; Cascinu, S.; Shchepotin, I.; Maurel, J.; Cunningham, D.; Tejpar, S.; Schlichting, M.; Zubel, A.; Celik, I.; Rougier, P.; Ciardiello, F. Cetuximab plus irinotecan, fluorouracil, and leucovorin as first-line treatment for metastatic colorectal cancer: updated analysis of overall survival according to tumor KRAS and BRAF mutation status. J. Clin. Oncol., 2011, 29 (15), 2011-2019.

[18] Tol, J.; Punt, C. J. Monoclonal antibodies in the treatment of metastatic colorectal cancer: a review. Clin. Ther., 2010, 32 (3), 437-453.

[19] SISTAR Available at: http://statistica.regione.veneto.it/sistar/inde x.jsp

[20] IOV. Available at: http://www.registrotumoriveneto.it/home.asp

[21] Lenz, H. J. Cetuximab in the management of colorectal cancer. Biologics, 2007, 1 (2), 77-91.
[22] Lidgren, M.; Wilking, N.; Jonsson, B.; Rehnberg, C. Costeffectiveness of HER2 testing and trastuzumab therapy for metastatic breast cancer. Acta. Oncol., 2008, 47 (6), 1018-10128.

[23] Drummond, M. F.; Sculpher, M. J.; Torrance, W. G.; O'Brien, B. J.; Stoddart, G. L. Methods. for the economic. Evaluation. of health. Care. Programmes, $3^{\text {rd }}$ ed., Oxford University Press: Oxford; New York, 2005; p. 379.

[24] Kumaraswamy, P A. generalized probability density function for double-bounded random processes. J. Hydrol, 1980, 46 (1-2), 7988.

[25] Van Hauwermeiren, M.; Vose, D.; Vanden Bossche, S. A Compendium of Distributions. Software, V., Ed. Ghent, Belgium, 2012. Available at: www.vosesoftware.com [Accessed on: $29^{\text {th }}$ May 2012].

[26] France, G.; Taroni, F. The evolution of health-policy making in Italy. J. Health. Polit. Policy. Law, 2005, 30 (1-2), 169-187.

[27] Ellis, L. M.; Hicklin, D. J. VEGF-targeted therapy: mechanisms of anti-tumour activity. Nat. Rev. Cancer, 2008, 8 (8), 579-591.

[28] Fragoulakis, V.; Papagiannopoulou, V.; Kourlaba, G.; Maniadakis, N.; Fountzilas, G. Cost-minimization analysis of the treatment of patients with metastatic colorectal cancer in Greece. Clin. Ther, 2012, 34 (10), 2132-2142.

[29] Hoyle, M. The effectiveness and cost-effectiveness of cetuximab (mono- or combination chemotherapy), bevacizumab (combination with (non-oxaliplatin chemotherapy) and panitumumab (monotherapy) for the treatment of metastatic colorectal cancer after first-line chemotherapy (review of technology appraisal 150 and part review of technology appraisal 118): a systematic review and economic model. Peninsula Technology Assessment Group (PenTAG) 2011.

[30] Hakulinen, T. Cancer Incidence in Five Continents Vol. VII. D. M. Parkin, S. L. Whelan, J. Ferlay, L. Raymond and J. Young (eds), IARC Scientific Publications No. 143, Lyon, 1997. No. of pages: xxxiv+1240. Price: £129. ISBN 928322143 5. Stat. Med., 2000, $19(9), 1261-1263$

[31] Pasetto, L. M.; Monfardini, S. Colorectal cancer screening in elderly patients: when should be more useful? Cancer. Treat. Rev., 2007, 33 (6), 528-532.

[32] Bracci, E.; Pizzo, E. An economic evaluation of a colorectal cancer screening programme. Translated from Italian: La valutazione economica di un programma sanitario di screening al colon-retto. 2007.

[33] Osservatorio Nazionale Screening. Screening del tumore del colon retto Iacobelli: Roma, 2011.

[34] Legge 23 dicembre 2000, n.388, art.85, co.4. Italiano, P., Ed. Gazzetta Ufficiale: 2000; vol. n. 302 del 29 dicembre 2000

[35] Ministri, P. d. C. d. Conferenza permanente per i rapport tra stato e regioni e le province autonome di Trento e Bolzano. 2005.

[36] Seifeldin, R.; Hantsch, J J. The economic burden associated with colon cancer in the United States. Clin. Ther., 1999, 21 (8), 1370-9.

[37] Zorzi, M.; Baracco, S.; Fedato, C.; Grazzini, G.; Sassoli De' Bianchi, P.; Senore, C.; Visioli, C. B.; Cogo, C. Screening for colorectal cancer in Italy, 2009 survey. Epidemiol. Prev., 2011, 35 (5-6 Suppl 5), 55-77.

[38] GISCOR, Piemonte. Screening del cancro colorettale, valutazione dei costi. Gruppo Italiano Screening Colorettale: Centro di Riferimento per l'Epidemiologia e la Prevenzione Oncologica in Piemonte Università degli Studi di Torino, vol. 9, 2005.

[39] Ngoma, T. World Health Organization cancer priorities in developing countries. Ann. Oncol., 2006, 17 Suppl 8, viii9-viii14. 